Copyright (C1996, American Institute of Aeronautics and Astronautics, Inc.

AIAA Meeting Papers on Disc, January 1996

A9618810, NSF CTS-95-03208, NGT-51070, AIAA Paper 96-0857

\title{
Direct numerical simulations of flows with phase change
}

\author{
D. Juric \\ Michigan Univ., Ann Arbor \\ G. Tryggvason \\ Michigan Univ., Ann Arbor
}

\section{AIAA 34th Aerospace Sciences Meeting and Exhibit, Reno, NV Jan 15-18, 1996}

We present results from direct numerical simulations of two types of phase-change processes: directional solidification of a binary alloy, and film boiling. We use a 2D finite-difference/front-tracking method which allows the evolution of the interface between the phases to be followed. The discontinuities in material properties between the phases, as well as topological changes, are easily handled. In directional solidification, the fully coupled solute and energy equations for a dilute binary alloy without fluid flow are solved. We demonstrate the evolution of a cellular interface with rejection of solute ahead of the advancing interface and in the intercellular grooves. The numerical results for the transition from a planar to a cellular interface are in excellent agreement with linear stability theory. The film boiling problem couples the phase change with fluid flow. We study the growth and dynamics of a vapor layer adjacent to an upward facing, flat, heated surface. Vaporization of the liquid at the liquid-vapor interface continually replenishes the vapor lost due to bubble departure from the interface. (Author) 


\title{
DIRECT NUMERICAL SIMULATIONS OF FLOWS WITH PHASE CHANGE
}

\author{
D. Juric and G. Tryggvason \\ Department of Mechanical Engineering \\ The University of Michigan \\ Ann Arbor, Michigan
}

\begin{abstract}
$\underline{\text { Abstract }}$
We present results from direct numerical simulations of two types of technologically important phase change processes: directional solidification of a binary alloy and film boiling. We use a two-dimensional finite-difference/front-tracking method which allows us to accurately follow the evolution of the interface between the phases. The method is general in the sense that discontinuities in material properties between the phases as well as topological changes are easily handled.

In the directional solidification problem the fully coupled solute and energy equations for a dilute binary alloy without fluid flow are solved. The effects of latent heat, unequal material properties between liquid and solid and unsteady effects are completely taken into account. We demonstrate the evolution of a cellular interface with rejection of solute ahead of the advancing interface and in the intercellular grooves. The numerical results for the transition from a planar to a cellular interface are in excellent agreement with linear stability theory.

The film boiling problem couples the phase change with fluid flow. This requires the solution of the Navier-Stokes and energy equations with interphase mass transfer. We study the growth and dynamics of a vapor layer adjacent to an upward facing, flat, heated surface. Vaporization of the liquid at the liquid-vapor interface continually replenishes the vapor lost due to bubble departure from the interface.
\end{abstract}

\section{Introduction}

Phase change plays a central role in energy systems and materials processes. However it is usually the combination of phase change with fluid flow and heat transfer that determines, for exam-

Copyright (C) 1996 by Damir Juric. Published by the American Institute of Aeronautics and Astronautics, Inc. with permission. ple, the efficacy of heat transfer equipment or the microstructure of solidified alloys. The electrical properties of semiconductors and the mechanical properties of single crystal turbine blades are closely coupled to fluid convection and processing conditions during directional solidification. Research on advanced materials and manufacturing processes such as rapid solidification or spray casting for the production of net or near-net shaped components depends on the ability to control and understand fluid flow during solidification. The power generation industry takes advantage of the high heat transfer rates associated with phase change in boiling to extract energy from solar, fossil and nuclear fuels. Designers of energy generation systems for spacecraft must deal with the added complication of handling low boiling point cryogenic fluids in the absence of gravity.

Experimental investigations of phase change in solidification and boiling are generally quite difficult due to the large range of important time and length scales involved. In addition, the harsh thermal and chemical environments in directional solidification furnaces make it nearly impossible to obtain measurements of crystal forming processes. Numerical simulations hold the promise to complement experimental investigations and provide information that is hard to measure. Although computations of industrial scale processes are currently out of reach, a fundamental understanding of the complex physics at the small scale can provide much needed insight to larger problems. By understanding this small scale information we hope to make progress toward the goal of providing quantitative predictions for linking operating conditions to large scale aspects of heat exchanger design and efficiency or to mechanical properties of solidified materials, for example.

We have recently developed a numerical technique for fluid flow with phase change that has allowed us to simulate problems with relatively complex motion of the boundary separating two (or more) fluids [1]. The method is based on a fi- 
nite difference approximation of the Navier-Stokes and energy equations and an explicit tracking of the phase boundary. The numerical solution of the full phase change problem with fluid flow is particularly difficult due to the coupling of the mass, momentum and energy transport with the interface dynamics and since interphase mass transfer results in discontinuous velocities at the phase boundaries. For problems without phase change the front-tracking method has been successfully used to investigate the collision of drops $[2,3]$, thermal migration of drops [4] and the evolution of several bubbles [5]. For problems without fluid flow we have used the method to simulate the dendritic solidification of pure materials as well as binary alloys $[6,7]$. In this paper we present results for two types of technologically important phase change processes. Section 2 deals with binary alloy solidification and and section 3 with film boiling.

\section{Directional Solidification of a Binary Alloy}

Directional solidification is one of several commercially important methods for solidifying metals. The basic idea in this process is to translate the part to be solidified from the hot to the cold end of a furnace. The translation velocity, $V_{o}$, and the temperature gradient in the furnace, $G$, along with the initial alloy composition, $C_{o}$, are the controllable process variables which determine the microstructure and the physical properties of the final solidified part. The liquid-solid interface during solidification is generally not planar but forms a cellular or dendritic structure depending on the processing conditions. Understanding and linking the behavior and geometry of the interface to the processing conditions is then of crucial importance to metallurgists. Until recently this understanding was mostly empirical. The modern science of metallurgy was pioneered by Chalmers and co-workers (see Rutter and Chalmers [8] and Tiller et al [9]) in the 1950's when they introduced the "constitutional supercooling" criterion for the onset of instability in a planar interface. This criterion is a simple and useful quantitative tool for producing a desired metallurgical microstructure. Mullins and Sekerka [10] refined this criterion by performing a linear stability analysis of the interface under more general conditions. For low solidification velocities, $V_{o}$, the Mullins-Sekerka instability criterion becomes equivalent to that of constitutional supercooling.

More recently, numerical work on the directional solidification problem has attempted to overcome the many simplifying assumptions of the previous analyses to provide more detailed quantitative predictions. Brown and co-workers [11]-[18] have developed and used boundary conforming finite element methods to study the growth and stability of solidification cells in detail. Wheeler et al [19], Boettinger et al [20] and Warren and Boettinger [21] have developed phase-field models for isothermal solidification of a binary alloy. A comprehensive review of methods for alloy solidification and morphological stability theory in general is provided by Coriell and McFadden, [22].

We use a new front-tracking method to solve the directional solidification problem. The fully coupled solute and energy equations for a dilute binary alloy are solved and the effects of latent heat, unequal material properties between liquid and solid and unsteady effects are completely taken into account.

\section{Formulation}

For the problem of solidification of a dilute binary alloy, we write the governing solute and energy equations for both the liquid and solid phases simultaneously. In writing this single field representation we carefully account for the effect of the interface between the phases and the jump in material properties from one phase to another. The densities of the liquid and solid phases are assumed to be equal and constant. Volume contraction and expansion, as well as fluid convection effects are thereby neglected. These effects are addressed in section 3 for the film boiling problem. The thermal conductivity, volumetric specific heat and chemical diffusivity of each phase are constant but not necessarily equal.

The energy equation is

$$
\frac{\partial}{\partial t}(c T)=\nabla \cdot K \nabla T+Q
$$

where $c$ is the volumetric specific heat, $K$ is the thermal conductivity and

$$
Q=\int_{\mathcal{A}} q \delta\left(\mathbf{x}-\mathbf{x}_{f}\right) d \mathcal{A}
$$

$\delta\left(\mathbf{x}-\mathbf{x}_{f}\right)$ is a three-dimensional delta function that is non-zero only at the interface where $\mathbf{x}=$ $\mathbf{x}_{f} \cdot q$ is the energy source due to liberation or absorption of the volumetric latent heat, $L$, at the interface

$$
q=L \mathbf{V} \cdot \mathbf{n} .
$$

$\mathbf{V}=\left(d \mathbf{x}_{f} / d t\right)$ is the interface velocity and $\mathbf{n}$ is the normal to the interface. In this equation $L$ takes into account unequal specific heats:

$$
L=L_{o}+\left(c_{1}-c_{2}\right) T_{m} .
$$


$L_{0}$ is the customary latent heat measured at the reference equilibrium melting temperature of the pure material, $T_{m}$. The subscript 1 will refer to the solid phase and 2 to the liquid phase.

The solute equation is usually written for both phases separately as

$$
\frac{\partial C_{1}}{\partial t}=\nabla \cdot D_{1} \nabla C_{1} \quad \text { and } \quad \frac{\partial C_{2}}{\partial t}=\nabla \cdot D_{2} \nabla C_{2}
$$

where $C$ is the solute concentration. The solute balance at the interface then requires that

$$
\left(D_{2} \nabla C_{2}-D_{1} \nabla C_{1}\right) \cdot \mathbf{n}=C_{2}(k-1) \mathbf{V} \cdot \mathbf{n} \text {. }
$$

where $k=C_{1}\left(\mathrm{x}_{f}(t)\right) / C_{2}\left(\mathrm{x}_{f}(t)\right)$ is the partition coefficient and is assumed to be constant.

We would like to recast Eqs.(5) and (6) in the form of a single equation for both phases. If we define new variables for the solute concentration and diffusivity (see acknowledgments)

$$
(\tilde{C}, \tilde{D})= \begin{cases}\left(C_{1} / k, k D_{1}\right), & \text { in the solid } \\ \left(C_{2}, D_{2}\right), & \text { in the liquid }\end{cases}
$$

then the single field representation is

$$
\frac{\partial \tilde{C}}{\partial t}=\nabla \cdot \tilde{D} \nabla \tilde{C}+S
$$

$\mathrm{S}$ is the source term which accounts for rejection or absorption of the solute at the interface

$$
S=\int_{\mathcal{A}} s \delta\left(\mathrm{x}-\mathrm{x}_{f}\right) d \mathcal{A} .
$$

and $s$ is the source of solute

$$
s=\tilde{C}_{f}(1-k) \mathbf{V} \cdot \mathbf{n}
$$

where $\tilde{C}_{f}=\tilde{C}\left(\mathbf{x}_{f}(t)\right)$ is the value of the transformed concentration at the interface. Note that the transformed concentration is continuous at the interface. Since the interface is tracked we can regain the original concentration and diffusivity fields from the known position of the interface by, for example,

$$
C=\tilde{C}+(k \tilde{C}-\tilde{C}) I(\mathbf{x}) .
$$

$I(\mathbf{x})$ is a material indicator function similar to the phase-field variable in phase-field models, that has the value 1 in phase 1 and 0 in phase 2. Unlike in phase-field methods we determine $I(\mathbf{x})$ from the known position of the tracked interface rather than using it to determine the position of the interface. This function also allows us to evaluate the values of the thermal conductivity and specific heat at every location by

$$
K(\mathbf{x})=K_{2}+\left(K_{1}-K_{2}\right) I(\mathbf{x})
$$

and similarly for the specific heat.

In addition to the governing equations, an interface condition on the temperature must be satisfied at the phase boundary [23]

$$
\begin{gathered}
T_{f}-T_{m}+\frac{T_{m}\left(c_{2}-c_{1}\right)}{L_{o}}\left[T_{f} \ln \frac{T_{f}}{T_{m}}+T_{m}-T_{f}\right] \\
+\frac{\sigma(\mathbf{n}) T_{m}}{L_{o}} \kappa-m \tilde{C}_{f}+\frac{\mathbf{V} \cdot \mathbf{n}}{\nu(\mathbf{n})}=0
\end{gathered}
$$

where $T_{f}=T\left(\mathbf{x}_{f}(t)\right)$ is the interface temperature, $\sigma(\mathbf{n})$ and $\nu(\mathbf{n})$ are the anisotropic surface tension and kinetic mobility respectively. $\kappa$ is twice the mean curvature which is positive when the center of curvature lies in the solid phase and $m$ is the slope of the liquidus line and is assumed to be a constant.

The numerical construction of the indicator function from the tracked interface and other details of the numerical method are described in detail in [1] for the solidification of pure materials. The binary alloy problem requires only minor, straightforward modifications to that method. Here, we only briefly outline the procedure. Equations (1) and (8), are discretized using a conservative, second order, centered difference scheme for the spatial variables. We use an explicit, first order, forward Euler time integration method for short time calculations and a second order time integration method for calculations that require accuracy over a longer time. The interface is represented by separate, non-stationary computational points connected to form a one-dimensional front which lies within the two-dimensional stationary mesh. The front is used to advect the discontinuous material property fields and to calculate interface curvature and normal velocity. Information is passed between the moving interface and the stationary grid using Peskin's immersed boundary technique [24].

\section{Results}

The simulations of directional solidification of a binary alloy are performed in a two-dimensional rectangular domain which is periodic in the $x$ direction. The upper-portion of the domain is initially liquid and is separated from the solid in the lower portion by the interface. The initial solidliquid interface is nearly planar with a small amplitude cosine perturbation. The specified temperature gradient at the top boundary in the liquid is 


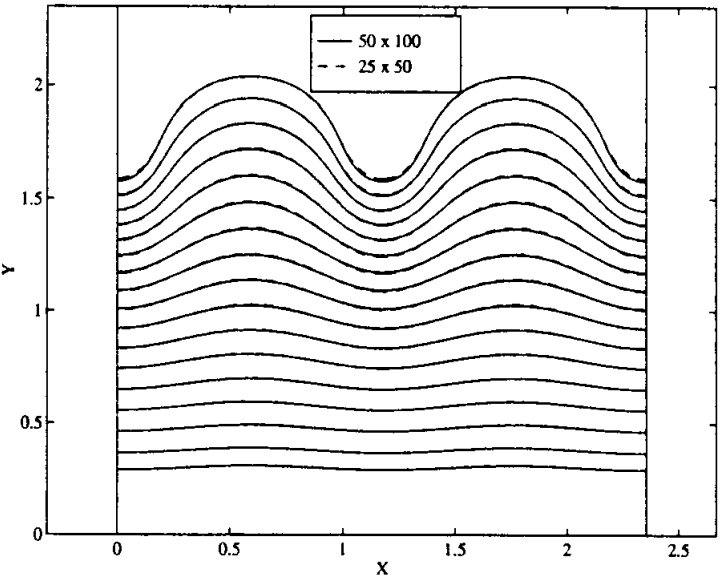

Figure 1: Growth of the Mullins-Sekerka instability in directional solidification of a binary alloy. Results from two different resolutions are superimposed. The coarse resolution performs almost identically to the finer resolution. The average interface velocity of our calculation and the growth rate of the interface perturbation amplitude match the linear theory for early times.

$G_{l}$ and at the bottom boundary in the solid is $G_{s}$. The initial temperature fields are linear in each phase

$$
\begin{aligned}
& T(x, y)=G_{s}\left(y-y_{f}\right)+T_{f} \text { in the solid, } \\
& T(x, y)=G_{l}\left(y-y_{f}\right)+T_{f} \text { in the liquid }
\end{aligned}
$$

where the initial interface temperature, $T_{f}$, is found from Eq.(13). The initial concentration field is $C_{o}$ in the solid and

$C(x, y)=C_{o}\left[1+\left(\frac{1-k}{k}\right) \exp \left(-\frac{V_{o}\left(y-y_{f}\right)}{D_{2}}\right)\right]$

in the liquid. This is simply the liquid phase solute concentration profile of a steadily advancing planar interface. The concentration gradients at the top and bottom boundary are specified to be zero.

The problem is governed by the following independent parameters:

$$
\begin{aligned}
& \begin{array}{llllll}
c_{1} & c_{2} & K_{1} & K_{2} & D_{1} & D_{2}
\end{array} \\
& L_{\circ} \sigma \nu T_{m} k \Delta T_{o} G_{s} G_{l} .
\end{aligned}
$$

Scaling length by $l_{d}=D_{2} / V_{o}$, time by $\tau=$ $D_{2} / V_{o}^{2}$, concentration by $C_{o}$ and temperature by $\Delta T_{o}$ the resulting 10 dimensionless parameters are

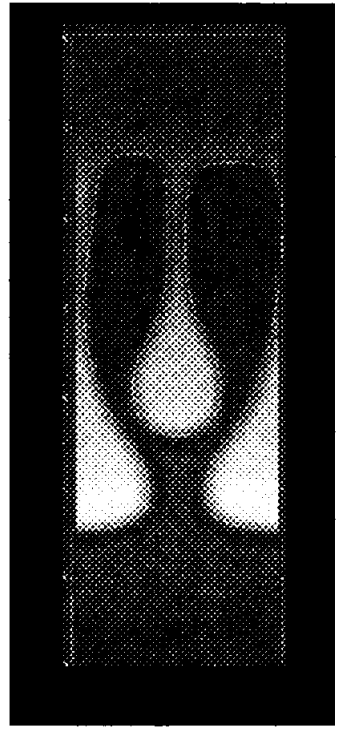

Figure 2: For a more unstable case the interface deforms quickly, bifurcates and begins to grow in a cellular pattern. The lighter shades of gray represent higher solute concentration and the white line is the interface. The rejection of solute ahead of the advancing interface and into the intercellular grooves is clearly visible.

the 3 ratios of material properties between liquid and solid, the Lewis Number, $L e=D_{2} c_{2} \rho_{2} / K_{2}$, the Stefan Number, $S t=c_{2} \Delta T_{o} / L_{o}$, the nondimensional temperature gradients at the top and bottom boundaries, $G_{l}^{*}=G_{l} l_{d} / \Delta T_{o}$ and $G_{s}^{*}=$ $G_{s} l_{d} / \Delta T_{o}$, the partition coefficient, $k$, the MullinsSekerka stability parameter, $A=k \sigma T_{m} / l_{d} L_{o} \Delta T_{o}$, and the nondimensional kinetic mobility, $B=$ $V_{o} / \nu \Delta T_{o}$. From the phase diagram geometry for dilute solutions and constant $m$ and $k$, we know that $\Delta T_{o}=m C_{o}(k-1) / k$.

The simulation in figure (1) was run with the following parameters

$$
\begin{gathered}
\frac{D_{2}}{D_{1}}=1000, \frac{K_{2}}{K_{1}}=1, \frac{c_{2}}{c_{1}}=1, \quad k=0.4 \\
L e=0.001, S t=0.01, \quad A=0.004 \quad B=0.002 \\
G_{l}^{*}=0.1 \quad G_{s}^{*}=0.2 .
\end{gathered}
$$

The computational domain is $\lambda \times 2 \lambda$ where $\lambda$ is the wavelength of the fastest growing unstable mode of the Mullins-Sekerka instability. For this case $\lambda=1.1768$. (Two periods are plotted in this figure for clearer illustration.) As the liquid solidifies the interface exhibits the growth of the MullinsSekerka instability. As a test of grid refinement, 
results from two different resolutions are superimposed. The coarse resolution performs almost identically to the finer resolution. Mullins and Sekerka neglected solid diffusion, interface kinetics and unsteady effects and assumed that the interface moves with a constant mean velocity. We make none of these assumptions in our numerical model. However for comparison, we choose parameters to approximately match the assumptions made in the linear theory. We would expect the best agreement early in the calculation before nonlinear effects come into play. The average interface velocity of our calculation and the growth rate of the interface perturbation amplitude match the linear theory for early times. As the interface deforms more greatly, these values drop below the theory.

Figure (2) shows a more unstable case. The interface deforms quickly, bifurcates and begins to grow in a pronounced cellular pattern. The lighter shades of gray represent higher solute concentration and the white line is the interface. The rejection of solute ahead of the advancing interface and into the intercellular grooves is clearly visible. For this case we have increased the Stefan number to 0.05 and introduced a four-fold anisotropy in the surface tension.

\section{Film Boiling}

In film boiling from an upward facing, flat surface a layer of vapor completely blankets the heated surface. The vapor is constantly depleted by break off and rise of vapor bubbles and is replenished by vaporization of liquid at the liquidvapor interface. The vapor layer acts as an insulator thereby lowering the heat transfer rate and increasing the heater surface temperature. In processes where the heat flux is the controllable variable such as in nuclear reactor operation or in electrically heated applications, exceeding the critical nucleate boiling heat flux can be dangerous. The process immediately jumps to the film boiling regime where heater damage can occur due to the high surface temperatures.

Efforts to understand the processes involved in boiling have focused mainly on simple numerical and analytical models of vapor bubble dynamics [25]-[32]. Due to the complexity of the full liquidvapor phase change problem, an assumed interface shape along with various assumptions concerning surface tension, fluid viscosity and vapor phase velocity and temperature are usually incorporated. The numerical solution of the full phase change problem with fluid flow is particularly difficult due to the coupling of the mass, momentum and energy transport with the interface dynamics and since interphase mass transfer results in discontinuous velocities at the phase boundaries. Welch [33] has made significant progress in using a twodimensional, moving mesh, finite volume method to solve the mass, momentum and energy equations for liquid-vapor flows with phase change. However, his method is restricted to flows with only small distortion of the liquid-vapor interface.

In a previous paper, we developed a general two-dimensional front-tracking method for liquidvapor flows with phase change that can handle large interface deformations and topology changes [1]. We use the method here to present calculations of film boiling, with the break off and rise of bubbles from an unstable liquid-vapor interface.

\section{Formulation}

We now broaden the scope of the phase change problem to include the solution of the NavierStokes and energy equations. As before we write the governing equations for both phases simultaneously. However we must now also take into account mass transfer across the interface and force as well as energy sources at the interface. Note that in two-phase flow, additional terms appear in these equations due to the phase change and the fact that the interface is no longer a material interface. The fluid velocity at the interface and the interface velocity are unequal. This single field, local instant formulation incorporates the interface jump conditions into the governing equations as sources which act only at the interface. Kataoka [34] shows that this single field representation is equivalent to the local instant formulations of Ishii [35] and Delhaye [36]. They formulate the phase change problem in terms of local instant variables for each phase with appropriate jump conditions at the moving phase interface. These local instant formulations form the basis for formulations using various types of averaging.

The momentum equation is written for the entire flow field and the forces due to surface tension are inserted at the interface as body forces which act only at the interface. In conservative form this equation is

$$
\begin{gathered}
\frac{\partial}{\partial t}(\rho \mathbf{u})+\nabla \cdot(\rho \mathbf{u u}) \\
=-\nabla P-\rho \mathbf{g}+\nabla \cdot \mu\left(\nabla \mathbf{u}+\nabla \mathbf{u}^{T}\right)+\mathbf{F} .
\end{gathered}
$$

The notation follows customary convention: $\mathbf{u}$ is the fluid velocity field, $P$ is the pressure, $\rho$ is the 
density and $\mu$ is the viscosity. $\mathbf{F}$ is a source term which accounts for forces acting on the interface

$$
\mathbf{F}=\int_{\mathcal{A}} \mathbf{f} \delta\left(\mathbf{x}-\mathbf{x}_{f}\right) d \mathcal{A} .
$$

$\mathrm{f}$ is the surface tension normal to the interface,

$$
\mathbf{f}=\sigma \kappa \mathbf{n}
$$

The conservation of mass equation for a fluid with a volume expansion at the interface due to phase change is

$$
\nabla \cdot \rho \mathbf{u}=M
$$

where

$$
M=\int_{\mathcal{A}} m \delta\left(\mathbf{x}-\mathbf{x}_{f}\right) d \mathcal{A}
$$

and $m$ accounts for mass transfer across the interface due to the phase change

$$
m=\left(\rho_{1}-\rho_{2}\right) \mathbf{V} \cdot \mathbf{n} .
$$

Note that this formulation of Eq.(17) is equivalent to the customary statement of the conservation of mass principle. The subscript 1 will refer to the vapor phase and 2 to the liquid phase.

The energy equation is

$$
\frac{\partial}{\partial t}(\rho c T)+\nabla \cdot(\rho \mathbf{u} c T)=\nabla \cdot K \nabla T+Q
$$

where

$$
Q=\int_{\mathcal{A}} q \delta\left(\mathbf{x}-\mathbf{x}_{f}\right) d \mathcal{A} .
$$

$q$ is the energy source due to liberation or absorption of latent heat, $L$, at the interface

$$
q=\rho_{1} L\left(\mathrm{~V}-\mathbf{u}_{1}\right) \cdot \mathbf{n} .
$$

$\mathbf{u}_{1}$ is the vapor phase fluid velocity at the interface. In this equation $L$ takes into account unequal specific heats:

$$
L=L_{o}+\left(c_{2}-c_{1}\right) T_{v} .
$$

$L_{0}$ is the customary latent heat measured at the reference equilibrium vaporization temperature, $T_{v}$.

The interface temperature condition that must be satisfied on the phase boundary [23] is

$$
\begin{aligned}
& T_{f}-T_{v}-\frac{\sigma T_{v}}{\rho_{2} L_{o}} \kappa+\frac{T_{v}}{L_{o}}\left(\frac{1}{\rho_{2}}-\frac{1}{\rho_{1}}\right)\left(P_{v}-P_{\infty}\right) \\
& -\left(c_{2}-c_{1}\right) \frac{T_{v}}{L_{o}}\left[T_{f} \ln \frac{T_{f}}{T_{v}}+T_{v}-T_{f}\right]=0, \quad(24)
\end{aligned}
$$

where $P_{\infty}$ and $P_{v}$ are the ambient pressure and the pressure at the interface in the vapor respectively. To complete the formulation we specify the material property fields using the material indicator function, $I(\mathbf{x})$, as discussed in the previous section.

It is important to note that integration of Eqs.(14), (17) and (20) across the interface directly yields the correct jump conditions in the local instant formulation for two-phase systems given by Delhaye [36] and Ishii [35] with the assumptions that the interface is thin and massless and that the bulk fluids are incompressible. However, we allow for volume expansion at the interface in the conservation of mass, Eq.(17), but neglect the volume expansion term in the constitutive shear relation in the momentum, Eq.(14). In the energy equation, viscous dissipation and kinetic energy contributions from the product of the fluid velocity at the interface and the interface velocity are neglected. Contributions to the energy equation from interface stretching are usually small compared with the latent heat and are neglected. Thermocapillary effects are also neglected.

The details of the finite difference/fronttracking method used to solve the above system of equations will not be discussed here but are provided in [1].

\section{Results}

In these simulations we follow the evolution of an unstable vapor layer below a liquid layer which is below another vapor layer. The computations are performed in a $10 \times 30$ box with a grid resolution of $50 \times 150$. The domain is periodic in the $x$-direction. To allow for vaporization we let fluid exit at the top boundary where we specify the ambient pressure to be zero. The temperature field is initially zero everywhere with a heat flux, $q_{w}$, applied to the rigid bottom wall.

The physics of the phase change problem is governed by

$\rho_{1}, \rho_{2}, \mu_{1}, \mu_{2}, c_{1}, c_{2}, K_{1}, K_{2}, L_{o}, \sigma, T_{v}, g, q_{w}$

If we scale temperature by $\rho_{1} L_{o} / \rho_{2} c_{2}$ and choose appropriate length and time scales $l_{d}=$ $\left(\mu^{2} / g \rho^{2}\right)^{1 / 3}$ and $\tau=\left(\mu / \rho g^{2}\right)^{1 / 3}$ respectively, the 9 resulting dimensionless parameters are the Prandtl number, $\mathrm{Pr}=c_{2} \mu_{2} / k_{2}$, the Bond Number, $B o=\left(g \mu^{4} / \rho \sigma^{3}\right)^{1 / 3}$, the nondimensional wall heat flux, $q^{*}=q_{w} \rho_{2} c_{2} l_{d} / K_{2} \rho_{1} L_{o}$, the Nusselt number, $N u=q_{w} l_{d} / K_{1}\left(T_{w}-T_{v}\right)$, and a capillary parameter, $d_{o}=\rho_{2} c_{2} \sigma T_{v} / \rho_{1}^{2} L_{o}^{2} l_{d}$. Note that 
Copyright (C1996, American Institute of Aeronautics and Astronautics, Inc.

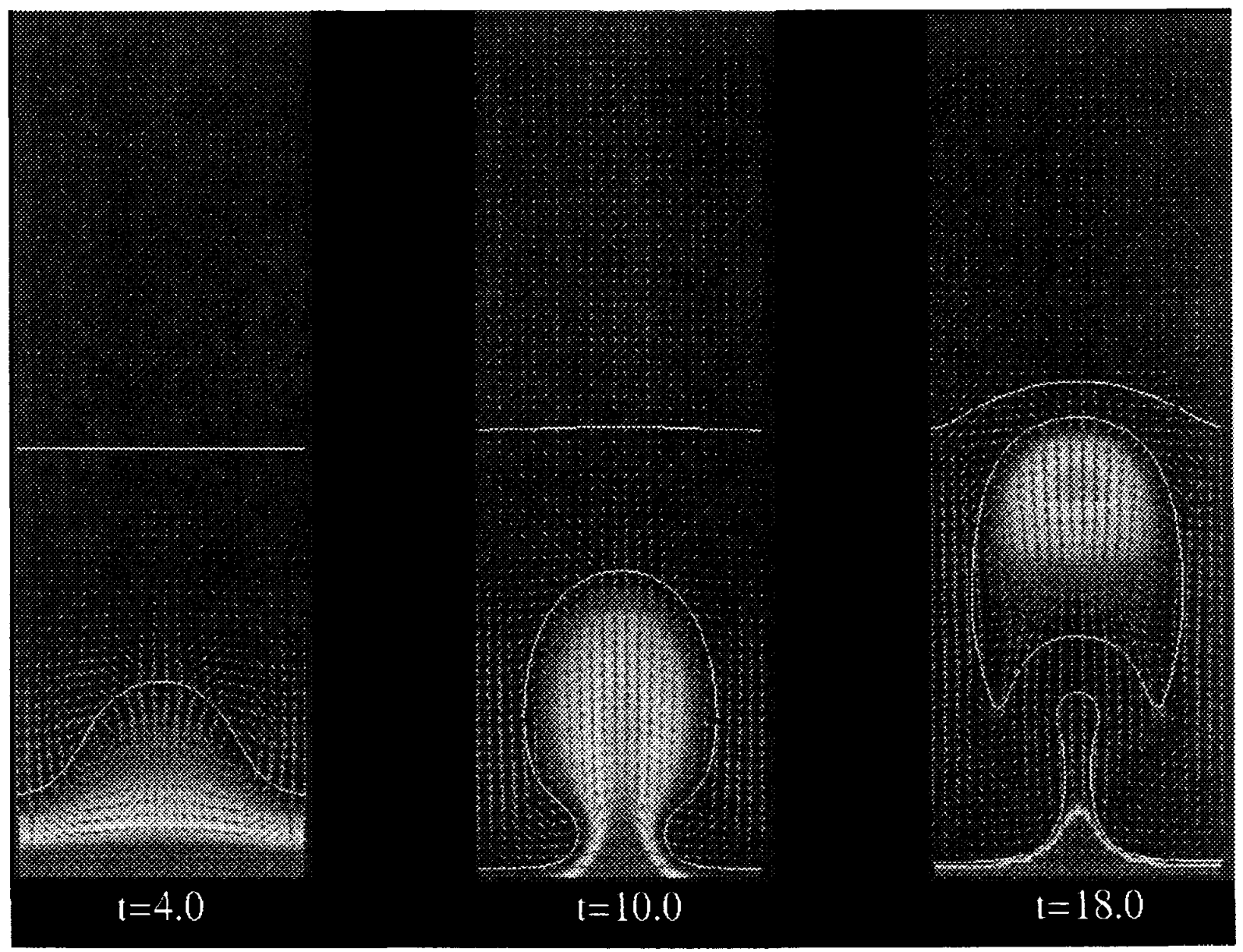

Figure 3: Film boiling simulation at three different times. The solid white lines are the liquid-vapor interfaces, the arrows represent velocity vectors and are plotted only at every fourth grid point. The temperatures are shown as shades of gray where the hottest regions are near the bottom wall and the coolest regions are in the liquid which remains nearly isothermal. A Rayleigh-Taylor instability forms with subsequent pinch off and rise of a vapor bubble. The bubble carries heated vapor up into the ambient liquid. The calculation is in a $10 \times 30$ box with grid resolution $50 \times 150 . \rho_{2} / \rho_{1}=10, \mu_{2} / \mu_{1}=$ $10, K_{2} / K_{1}=10, c_{2} / c_{1}=1, \quad \operatorname{Pr}=1, \quad q_{w}=0.5, \quad B_{o}=1, \quad d_{o}=0.002$ 


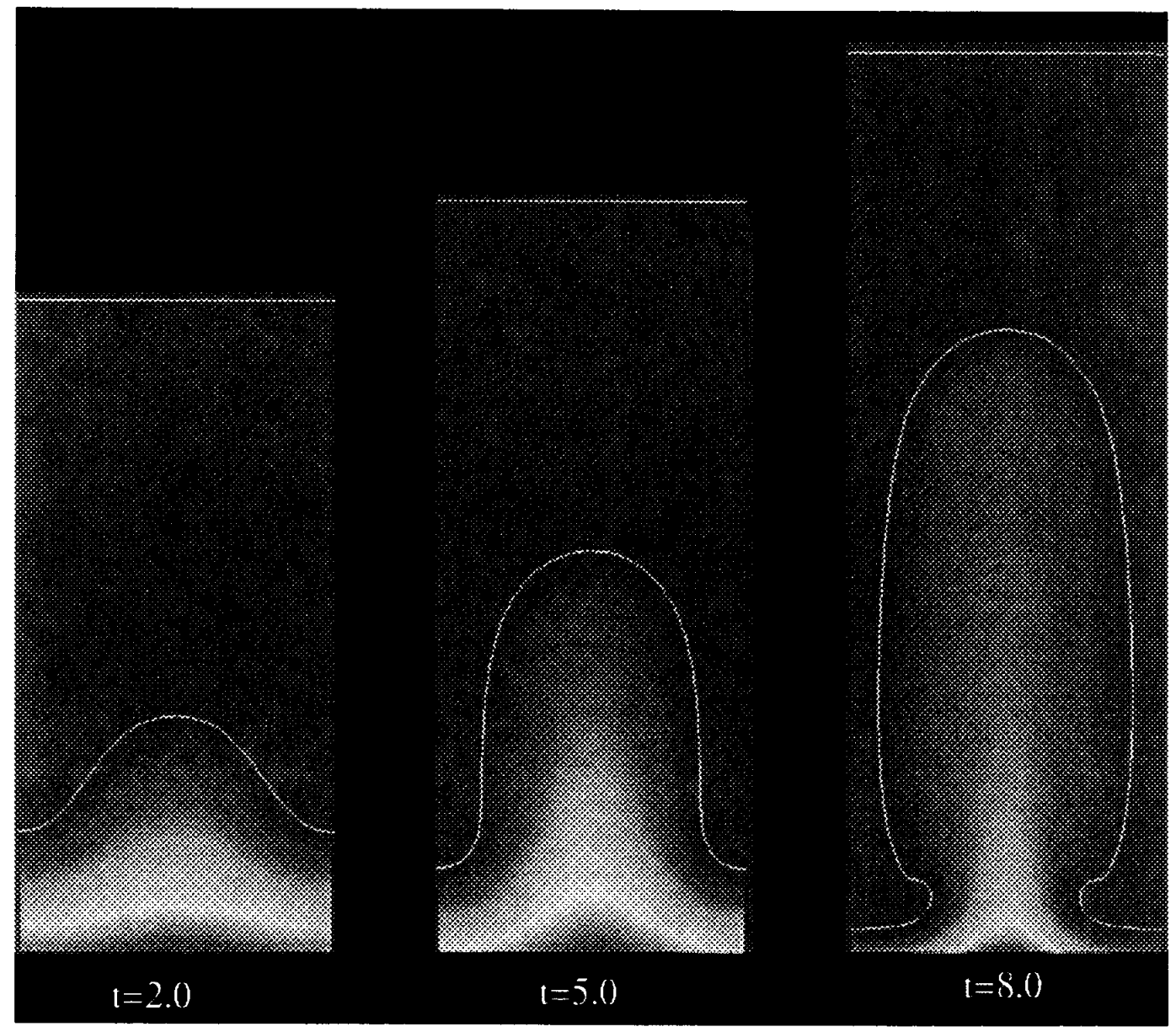

Figure 4: Film boiling simulation for three different times with the density of the liquid 100 times that of the vapor. The amount of vapor in the growing bubble near the bottom wall increases due to vaporization of liquid at the lower interface. Since the low density vapor takes up more volume, the fluid above it is pushed upwards and the upper interface moves passively upward with the fluid. The parameters are the same as in figure (3) except $\rho_{2} / \rho_{1}=100, \mu_{2} / \mu_{1}=40, K_{2} / K_{1}=20, q_{w}=10, B_{0}=0.05$. 


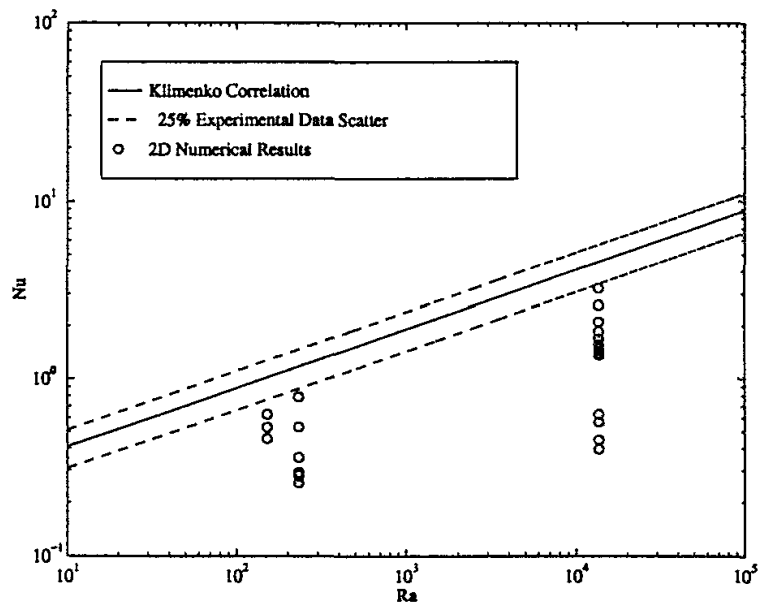

Figure 5: Comparison of heat transfer results from two-dimensional numerical simulations against a correlation by Klimenko [37]. For each of the three numerical runs at three different Rayleigh numbers the average Nusselt number along the heated bottom wall is plotted at different times. Two-dimensional simulations are in good agreement but consistently lower than 3-D experiments. Higher 3-D values are expected since heat flow is not confined to a plane and on average the height of the vapor layer above the heated wall would be lower and thus the heat transfer would be higher in $3-\mathrm{D}$ than in 2-D.

the Nusselt number is not set beforehand but is determined by the calculation of the wall temperature, $T_{w}$. In addition to these we must specify the 4 ratios of the material properties between the liquid and the vapor.

The simulation in figure (3) was run with the following parameters:

$$
\begin{aligned}
& \frac{\rho_{2}}{\rho_{1}}=10, \frac{\mu_{2}}{\mu_{1}}=10, \frac{K_{2}}{K_{1}}=10, \frac{c_{2}}{c_{1}}=1, \\
& \operatorname{Pr}=1, \quad q_{w}=0.5, \quad B_{o}=1, \quad d_{o}=0.002
\end{aligned}
$$

and is shown for three different times. The interfaces are plotted as the solid white lines while the arrows represent velocity vectors and are plotted only at every fourth grid point. The temperatures are shown as shades of gray where the hottest regions are near the bottom wall and the coolest regions are in the liquid which remains nearly isothermal. In the first frame of the figure the liquid vapor interface begins to exhibit a Rayleigh-Taylor instability with the formation of counterrotating vortices. Cold liquid is forced down toward the bottom wall and hot vapor is pushed up into the forming bubble. The interface then pinches together and in the last frame the separated bubble rises toward the upper interface carrying with it some of the hot vapor from the heated wall.

Vaporization of the liquid at the liquid-vapor interface continually replenishes the vapor lost due to bubble departure. This is seen more easily in the simulation shown in figure (4) where the density ratio and the wall heat flux are higher. The parameters are the same as in the previous figure except

$$
\begin{gathered}
\frac{\rho_{2}}{\rho_{1}}=100, \frac{\mu_{2}}{\mu_{1}}=40, \frac{K_{2}}{K_{1}}=20 \\
q_{w}=10, \quad B_{o}=0.05 .
\end{gathered}
$$

The amount of vapor in the growing bubble near the bottom wall is continually increasing due to vaporization of liquid at the lower interface. Since the low density vapor takes up more volume, the fluid above it is pushed upwards. Note that there is no vaporization at the upper interface. This interface simply moves passively upward with the fluid on either side of it. The fact that the upper interface does move upward is a good indicator that mass transfer is taking place across the lower interface.

In figure (5) we compare heat transfer results from our two-dimensional simulations against a correlation by Klimenko [37] on a plot of the Nusselt vs. Rayleigh numbers. Klimenko found that his correlation holds within $25 \%$ for many different fluids. The open circles represent the numerical results for three different runs at three different Rayleigh numbers. For each of the three runs the average Nusselt number along the heated bottom wall is plotted at different times. Thus we see a range of values for one run which represents the increasing and decreasing heat transfer from the wall as the height of the vapor layer adjacent to the wall decreases or increases with time. The experimental results would naturally give values averaged over the heated area as well as averaged in time. Our 2-dimensional numerical results are consistently lower than the values from 3-dimensional experiments. However we would expect our twodimensional results to exhibit lower heat transfer for several reasons. In three dimensions the heat flow is not confined to a plane. There are also more bubbles rising from many various points on a heated surface. On average the height of the vapor layer above the heated wall would be lower 
and thus the heat transfer would be higher in 3-D than in 2-D.

\section{Conclusions}

We have shown results from two-dimensional direct numerical simulations of directional solidification of a binary alloy and film boiling from a heated surface. Although these problems represent vastly different physics, they are both amenable to solution using a finite difference/front-tracking method. For the alloy solidification problem we demonstrate the growth of an unstable liquid-solid interface with solute rejection in the intercellular grooves. The results match the linear theory of Mullins and Sekerka for small interface deformations. For larger growth rates, the interface bifurcates and grows into deep cells with solute rich cellular grooves. In the film boiling problem we demonstrate the break off and rise of a bubble from an unstable liquid-vapor interface. The heat transfer results are in good agreement with experimental results within the limitations of the two-dimensional calculations.

\section{Acknowledgments}

This work was supported in part by NSF Grant CTS-9503208 and by NASA Graduate Student Fellowship NGT-51070. D.J. would like to acknowledge helpful discussions with Bruce Murray, Sam Coriell and Geoffrey McFadden of the National Institute of Standards and Technology regarding the formulation and numerical implementation of the alloy solidification problem.

\section{References}

[1] D. Juric and G. Tryggvason, "A FrontTracking Method for Liquid-Vapor Phase Change," in Advances in Numerical Modeling of Free Surface and Interface Fluid Dynamics, edited by P. E. Raad, T. T. Huang and G. Tryggvason, FED-Vol. 234, (ASME, New York, 1995) pp. 141-148.

[2] M.R. Nobari, Y.-J. Jan and G. Tryggvason, "Head on Collision of Drops - A Numerical Investigation," submitted to Phys. of Fluids $A$, (1993).

[3] M.R. Nobari and G. Tryggvason, AIAA 940835, "Numerical Simulations of Drop Collisions."
[4] S. Nas and G. Tryggvason, "Computational Investigation of Thermal Migration of Bubbles and Drops," in Fluid Mechanics Phenomena in Microgravity, edited by D.A. Siginer, R.L. Thompson and L.M. Trefethen, FEDVol. 175, (ASME, New York, 1993), pp. 7183.

[5] A. Esmaeeli, E.A. Ervin and G. Tryggvason, "Numerical Simulations of Rising Bubbles and Drops," to appear in Proceedings of the IUTAM Conference on Bubble Dynamics and Interfacial Phenomena, edited by J.R. Blake.

[6] D. Juric and G. Tryggvason, "A FrontTracking Method for Dendritic Solidification," J. Comp. Phys, 123, pp. 1-22 (1996).

[7] D. Juric and G. Tryggvason, "Directional Solidification of Binary Alloys using a FrontTracking Method," in preparation.

[8] J. W. Rutter and B. Chalmers, "A Prismatic Substructure Formed during Solidification of Metals," Can. J. Phys., 31, pp. 15-39 (1953).

[9] W. A. Tiller, K. A. Jackson, J. W. Rutter and B. Chalmers, "The Redistribution of Solute Atoms during the Solidification of Metals," Acta Metall., 1, pp. 428-437, (1953).

[10] W. W. Mullins and R. F. Sekerka, "Stability of a Planar Interface During Solidification of a Dilute Binary Alloy," J. Appl. Phys., 35, pp. 444-451 (1964).

[11] H. M. Ettouney and R. A. Brown, "FiniteElement Methods for Steady Solidification Problems," J. Comp. Phys., 49, pp. 118-150 (1983).

[12] L. H. Ungar and R. A. Brown, "Cellular Interface Morphologies in Directional Solidification. The One-Sided Model," Phys. Rev. B, 29, pp. 1367-1380 (1984).

[13] L. H. Ungar, M. J. Bennett and R. A. Brown, "Cellular Interface Morphologies in Directional Solidification III. The Effects of Heat Transfer and Solid Diffusivity," Phys. Rev. B, 31, pp. 5923-5930 (1985).

[14] L. H. Ungar and R. A. Brown, "Cellular Interface Morphologies in Directional Solidification IV. The Formation of Deep Cells," Phys. Rev. B, 31, pp. 5931-5940 (1985). 
[15] N. Ramprasad, M. J. Bennett and R. A. Brown, "Wavelength Dependence of Cells of Finite Depth in Directional Solidification," Phys. Rev. B, 38, pp. 583-592 (1988).

[16] M. J. Bennett and R. A. Brown, "Cellular Dynamics during Directional Solidification: Interaction of Multiple Cells," Phys. Rev. B, 39, pp. 11705-11723 (1989).

[17] K. Tsiveriotis and R. A. Brown, "BoundaryConforming Mapping Applied to Computations of Highly Deformed Solidification Interfaces," Int. J. Num. Meth. Fluids, 14, pp. 981-1003 (1992).

[18] K. Tsiveriotis and R. A. Brown, "Solution of Free-Boundary Problems Using FiniteElement/Newton Methods and Locally Refined Grids: Application to Analysis of Solidification Microstructure," Int. J. Num. Meth. Fluids, 16, pp. 827-843 (1993).

[19] A. A. Wheeler, W. J. Boettinger and G. B. McFadden, "Phase-Field Model for Isothermal Phase Transitions in Binary Alloys," Phys. Rev. A, 45, pp. 7424-7439 (1992).

[20] W. J. Boettinger, A. A. Wheeler, B. T. Murray and G. B. McFadden, "Prediction of Solute Trapping at High Solidification Rates Using a Diffuse Interface Phase-Field Theory of Alloy Solidification," Mat. Sci. Eng., A178, pp. 217-223 (1994).

[21] J. A. Warren and W. J. Boettinger, "Prediction of Dendritic Growth and Microsegregation Patterns in a Binary Alloy Using the Phase-Field Method," Acta Metall., 43, pp. 689-703 (1995).

[22] S. R. Coriell and G. B. McFadden, "Morphological Stability," in Handbook of Crystal Growth, Vol. 1, edited by D. T. J. Hurle (North-Holland, Amsterdam, 1993), pp.785857.

[23] V. Alexiades and A. D. Solomon, Mathematical Modeling of Melting and Freezing Processes (Hemisphere, Washington, DC,) 1993, p. 92 .

[24] C. S. Peskin, "Numerical Analysis of Blood Flow in the Heart," J. Comput. Phys., 25, 220-252 (1977).

[25] Lord Rayleigh, "On the Pressure Developed in a Liquid During the Collapse of a Spherical Cavity," Phil. Mag., 34, 94-98 (1917).
[26] F. Bosnjakovic, Tech. Mech. Thermodynamics, 1, 358 (1930).

[27] M.S. Plesset and S.A. Zwick, "A Nonsteady Heat Diffusion Problem with Spherical Symmetry," J. Appl. Phys., 23, 95 (1952).

[28] B.B. Mikic, W.M.Rohsenow and P. Griffith, "On Bubble Growth Rate," Int. J. Heat and Mass Transfer, 13, 657-666 (1970).

[29] M. Dalle Donne and M.P. Ferranti, "The Growth of a Vapor Bubble in Superheated Sodium," Int. J.Heat and Mass Transfer, 18, 477-493 (1975).

[30] H.S. Lee and H. Merte, "Spherical Bubble Growth in Liquids with Initial Uniform Superheat," Report No. UM-MEAM-93-06, Dept. of MEAM, U. of Michigan, July 1993, to Nasa Lewis Research Center, Cleveland, OH, NASA Contract No. NAS3-25812.

[31] R. C. Lee and J. E. Nydahl, "Numerical Calculation of Bubble Growth in Nucleate Boiling from Inception through Departure," $J$. Heat Transfer, 111, pp. 474-479 (1989).

[32] R. K. Patil and J. Prusa, "Numerical Solutions for Asymptotic, Diffusion Controlled Growth of a Hemispherical Bubble on an Isothermally Heated Surface," in Experimental/Numerical Heat Transfer in Combustion and Phase Change, edited by M. F. Modest, T. W. Simon and M. Ali Ebadian, HTD-Vol. 170 (ASME, New York, 1991).

[33] S. W. J. Welch, "Local Simulation of TwoPhase Flows Including Interface Tracking with Mass Transfer," J. Comp. Phys., 121, pp. 142-154 (1995)

[34] I. Kataoka, "Local Instant Formulation of Two-Phase Flow", Technical Report Institute of Atomic Energy, Kyoto University, Kyoto, Japan, Vol. 203, pp. 1-33 (1985).

[35] M. Ishii, Thermo-Fluid Dynamic Theory of Two-Phase Flow, Eyrolles, Paris, 1975.

[36] J.M. Delhaye, "Jump Conditions and Entropy Sources in Two-Phase Systems. Local Instant Formulation," Int. J. Multiphase Flow, 1, 395-409 (1974).

[37] V.V. Klimenko, "Film Boiling on a Horizontal Plate - New Correlation," Int. J. Heat and Mass Transfer, 24, pp. 69-79, (1981). 